\title{
Atomic Structure of Superconducting Tunnel Junctions using STEM and APT
}

\author{
Edwin Supple ${ }^{1}$, Megan Holtz ${ }^{1}$, Christopher J. K. Richardson ${ }^{2}$ and Brian Gorman ${ }^{1}$ \\ ${ }^{1}$ Colorado School of Mines, United States, ${ }^{2}$ Laboratory for Physical Sciences, United States
}

Superconducting qubits are a promising design for implementing large scale quantum computing. Current qubits, however, suffer from short decoherence times due in part to coupling with two level systems in and around the qubit devices. Most superconducting qubits use an amorphous $\mathrm{AlOx}$ barrier between polycrystalline aluminum superconductors to create the junction. Amorphous materials host numerous two level systems (TLS) and the amorphous AlOx barrier may limit qubit performance [1]. Nitrides can have low microwave loss and may allow fully epitaxial growth, reducing TLS density [2]. NbN-AlN-NbN qubits have been previously grown epitaxially on $\mathrm{MgO}$, a lossy substrate that is incompatible with quantum circuits [3]. Here we study NbTiN-AlN-NbTiN stacks using scanning transmission electron microscopy (STEM) and atom probe tomography (APT) to characterize the material phase, crystal orientation, defect chemistry, and barrier thickness. STEM and APT together provide nanoscale 3D visualization of the composition, defect chemistry, and phases at the buried interfaces, providing key feedback on their short decoherence times.

The NbTiN-AlN-NbTiN devices studied are grown via plasma-assisted molecular beam epitaxy onto c-plane oriented sapphire, with nominal layer thicknesses of $83 \mathrm{~nm}, 2 \mathrm{~nm}$, and $100 \mathrm{~nm}$ from bottom to top. APT specimens are prepared from the whole stack using standard FIB techniques onto tungsten half grids. STEM images and 4-D diffraction data are recorded of the specimens before and after APT analyses in order to document the specimen volume, crystallographic orientations, and interatomic spacings.

APT specimens nominally $150 \mathrm{~nm}$ in diameter were analyzed in a Cameca LEAP 4000 XSi using laser pulsing at $8 \mathrm{pJ}$ and $45 \mathrm{~K}$ base temperatures. Both nitrides studied and the sapphire substrate field evaporate as compounds even at low laser energies (Figure 1). NbN, TiN, TiN2, AlN, Al2N, AlN2, AlO, Al2O, Al3O2, $\mathrm{Al} 2 \mathrm{O} 2, \mathrm{Al} 2$, and $\mathrm{N} 2$ are detected as well as individual ions of $\mathrm{Nb}, \mathrm{Ti}, \mathrm{Al}, \mathrm{O}$, and $\mathrm{N}$. The resulting mass spectrum has significant overlaps. Most notable are $\mathrm{TiN}++, \mathrm{O} 2+$, and $\mathrm{Nb}+++$ at $\mathrm{m} / \mathrm{q}=31-33$; $\mathrm{Ti}++, \mathrm{Nb}++++$, and $\mathrm{A} 12 \mathrm{O}+++$ at $\mathrm{m} / \mathrm{q}=23-23.5$; and $\mathrm{Ti}+++, \mathrm{O}+$, and $\mathrm{O} 2++$ at $\mathrm{m} / \mathrm{q}=16$. Fortunately, oxygen containing species appear only in the substrate and at its interface. Two approaches are applied to evaluate the interfacial compositions and defect chemistries. In single-phase regions, ranges are applied only using elements that should be present in the region and deconvolution is used to further refine bulk compositions. At the NbTiNAIN interface, overlapping peaks are discarded entirely and only unique peaks are used to range the collected ions. Ion counts from the NbTiN ranged peaks are multiplied by adjustment factors to match chemistries and counts previously determined in the single-phase regions to account for unranged overlapping ions. Al and its nitrides do not overlap with the remainder of the mass spectrum.

The $\sim 2 \mathrm{~nm}$ thick AlN tunnel barrier was grown under non-optimized conditions and analyzed separately from the superconducting contacts. AlN layer thickness was found to vary by nearly the entire thickness within a 10 $\mathrm{nm}$ lateral region (Figure 2). Maximum Al content also varies from 27-36 atomic \% within the same $10 \mathrm{~nm}$ region. 4-D STEM diffraction data indicates that the AIN has a wurtzite crystal structure.

Models of amorphous TLS proposed in the literature suggest that point defects can act as origins for qubit decoherence. Since the APT contains both anion and cation chemical composition information, point defect reactions can be determined and free charges quantified on the nanometer scale. Both NbTiN layers are cation deficient in the bulk material, with $\mathrm{N}$ at least 52 atomic \% concentration. $\mathrm{Nb}$ and $\mathrm{Ti}$ vacancies are expected at 
the $\mathrm{NbTiN}-\mathrm{AlN}$ interface, assuming $100 \% \mathrm{~N}+$ at $\mathrm{m} / \mathrm{q}=14$. Any portion of $\mathrm{N} 2++$ would increase total $\mathrm{N}$ concentration in the NbTiN regions.

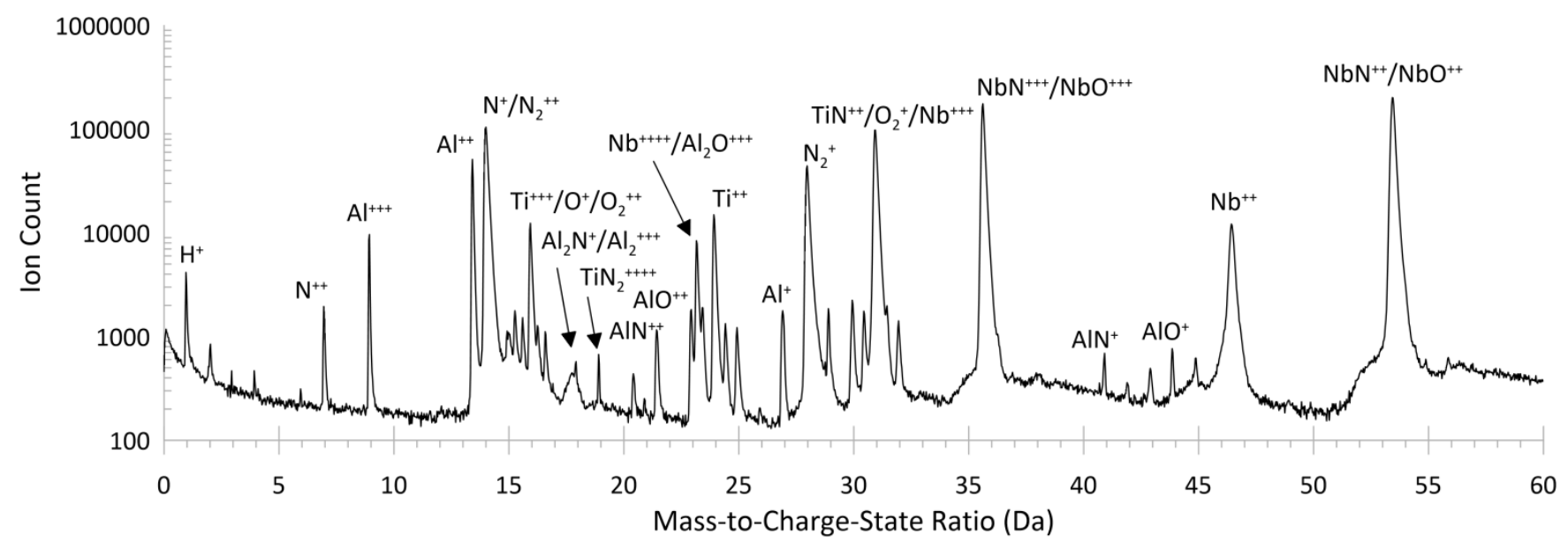

Figure 1. Representative mass spectrum from NbTiN-AIN-NbTiN on sapphire substrate, showing numerous peak overlaps, particularly at $\mathrm{m} / \mathrm{q}=16,23$, and 31 .

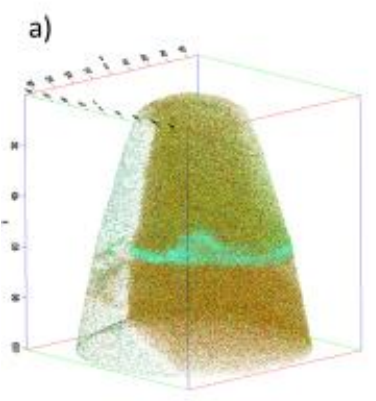

b)

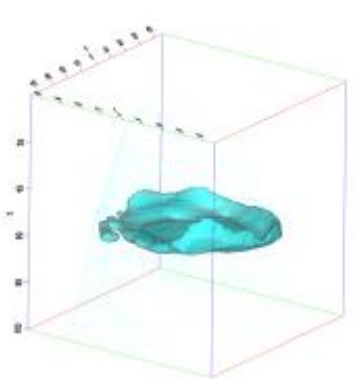

c)

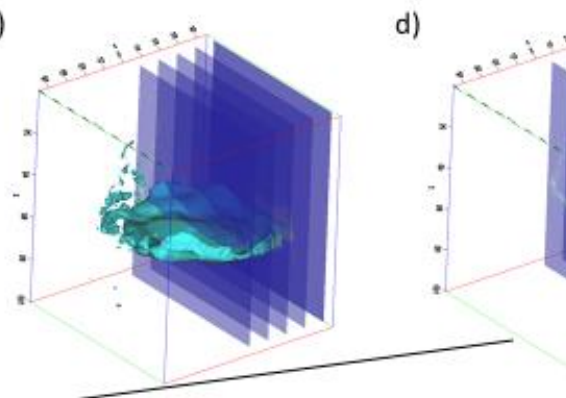

\section{(1)}

e)

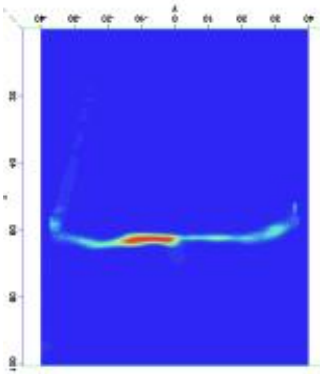

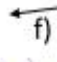

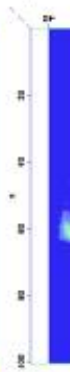

g)

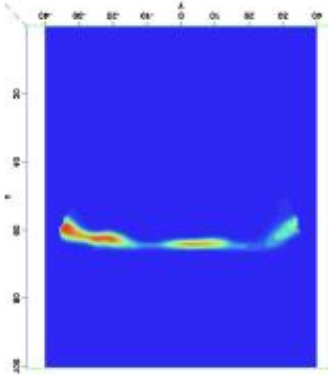

h)

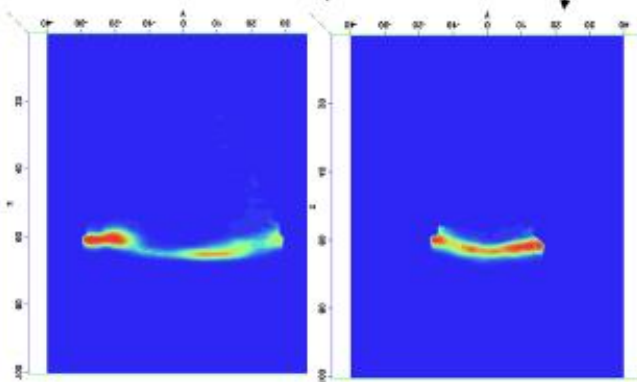

Figure 2. a) atom probe reconstruction showing $\mathrm{Al}$ ions in blue. b) $\mathrm{Al}$ isosurface superimposed over $\mathrm{Al}$ ion locations in reconstruction. c) $10 \mathrm{~nm}$ thick rectangular slices through $\mathrm{Al}$ isosurface. d) rectangular slices through $\mathrm{Al}$ isosurface, showing $\mathrm{Al}$ concentration within slice. e-i) 2D contour plots showing $\mathrm{Al}$ concentration in each slice. Note the variation in thickness and maximum concentration both in $\mathrm{x}$ plane within slices and between slices. 


\section{References}

[1] C. Müller, J. Lisenfeld, A. Shnirman, and S. Poletto, "Interacting two-level defects as sources of fluctuating high-frequency noise in superconducting circuits," Phys. Rev. B, vol. 92, no. 3, p. 035442, Jul. 2015, doi: 10.1103/PhysRevB.92.035442.

[2] C. J. K. Richardson, A. Alexander, C. G. Weddle, B. Arey, and M. Olszta, "Low-loss superconducting titanium nitride grown using plasma-assisted molecular beam epitaxy," J. Appl. Phys., vol. 127, no. 23, p. 235302, Jun. 2020, doi: 10.1063/5.0008010.

[3] Y. Nakamura, H. Terai, K. Inomata, T. Yamamoto, W. Qiu, and Z. Wang, "Superconducting qubits consisting of epitaxially grown NbN/AlN/NbN Josephson junctions," Appl. Phys. Lett., vol. 99, no. 21, p. 212502, Nov. 2011, doi: 10.1063/1.3663539.

[4] This work was funded by the Laboratory for Physical Sciences under contract \#H98230-19-C-0428. 